\title{
Silsesquioxane-Polythiophene Hybrid Copolymer as an Efficient Modifier for Single-Walled Carbon Nanotubes
}

\author{
Shuxi Gao $(\mathbb{D}$, Xiaoyong Hu, Lei Zhang, Yuliang Mai, Hao Pang, Yongqiang Dai, \\ and Bing Liao \\ Guangdong Provincial Key Laboratory of Industrial Surfactant, Guangdong Research Institute of Petrochemical and Fine \\ Chemical Engineering, Guangzhou, 510000 Guangdong, China \\ Correspondence should be addressed to Shuxi Gao; gaoshuxi@163.com and Bing Liao; liaobing@gic.ac.cn
}

Received 8 October 2019; Accepted 30 December 2019; Published 11 March 2020

Guest Editor: Feng Wu

Copyright (c) 2020 Shuxi Gao et al. This is an open access article distributed under the Creative Commons Attribution License, which permits unrestricted use, distribution, and reproduction in any medium, provided the original work is properly cited.

\begin{abstract}
One silsesquioxane-polythiophene hybrid copolymer, with combined star-like structure and intramolecular heterogeneity, was synthesized and sufficiently characterized via various methods, including FTIR, NMR, and SEC measurements. According to the exploration and characterization results, it was much more efficient at modifying SWNTs than its linear analogs in aqueous solution. The hydrophobic silsesquioxane core and PEDOT chains could locally anchor to the surface of the nanotubes, while the soluble flexible copolymer chains extended into the solution and rigid conjugated chains provided some $\pi$ - $\pi$ stacking effect to enhance adhesive force with the conjugated structure of the carbon nanotube, imparting steric stabilization to nanotube dispersion. The noncovalent interaction with SWNTs and solubility in aqueous solution improved the electrochemical characteristics of the modified-SWNT composite and availed for the preparation of a flexible and transparent electroactive film. Accordingly, this kind of silsesquioxane-polythiophene hybrid copolymer will be forwarded to apply to the assembling of flexible optoelectronic devices.
\end{abstract}

\section{Introduction}

Carbon nanotubes (CNTs) classified as multiwalled nanotubes (MWNTs) and single-walled nanotubes (SWNTs), upon the wrapped amount of a graphite sheet, have been used in multiple fields, such as composites, optoelectronic devices, field emission display, and energy storage, due to their unique physical, chemical, and structural properties [1-3]. However, the poor solubility and dispersibility of CNTs in both aqueous and organic media are major barriers for CNT applications, especially for SWNTs, as a result of the strong Van der Waals interaction between CNTs [4-8].

In order to obtain fine dispersion of CNTs in aqueous solution, various approaches that primarily paid attention on dispersion and dissolution properties have been developed, including covalent surface and noncovalent surface functionalization strategies [9-14]. Noncovalent modification has received considerable attraction for the modification of surface properties with no damage to the $\mathrm{sp} 2$ structure of the nanotube surface, involving $\pi-\pi$ interaction, hydrogen bonds, and ionic interaction by employing micromolecule surfactants or amphiphilic copolymers as modifiers.

In previously published works [15-17], copolymeric structures have the advantages of promoting CNTs' dispersibility in a wide range of solvents depending on the great affinity of hydrophobicity of the copolymers for the nanotube, and it has been demonstrated in several literatures that various polymers have effectively enhanced the long-term dispersion stability of CNTs through different types of interactions. Furthermore, the versatile chemistry of copolymer modifiers allows for tailoring and functionalizing polymer/nanotube composites with finely various properties, enhancing the application of CNTs, especially in fabricating carbon-based flexible transparent electrodes [18-21].

Herein, a novel star-shaped copolymer is reported to stabilize single-walled carbon nanotubes (SWNTs) in aqueous solution through noncovalent interactions. In the previous work of our team [4], we had adequately demonstrated that an octopus-shaped amphiphilic copolymer could generate a stable and uniform physical adsorption layer on a nanotube, 


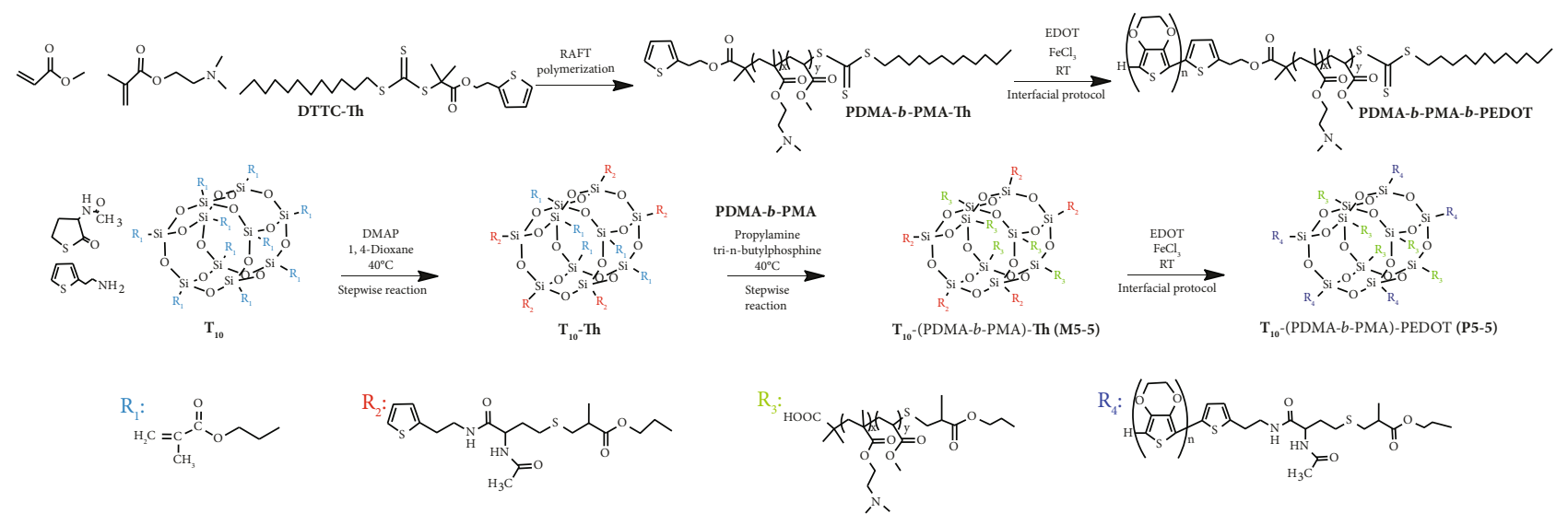

Scheme 1: Synthesis route to linear copolymers and star-shaped copolymers.

yielding much better efficiencies toward functionalization and dispersion, and multiple binding sites could assemble larger polymer aggregation that locally anchored onto the nanotube surface compared to its linear analogs that sequentially reduced the hydrophobicity of the sidewall surface for minimizing the connection with water and other tubes.

Therefore, we were motivated to extend SWNT modification to a silsesquioxane- (SQ-) cored star-like conjugated copolymer. Firstly, SQ would be convenient to constitute the core of star polymers. Secondly, the hydrophobic silsesquioxane cage could impart to amphiphilic copolymers' hydrophobic-hydrophobic interactions with CNTs [4]. Finally, the conjugated segments could generate $\pi-\pi$ interaction between conjugated chains and the hybridized CNT surface [22-24].

Herein, we report a star-like conjugated copolymer $\mathbf{T}_{\mathbf{1 0}^{-}}$ (PDMA-b-PMA)-PEDOT (P5-5) standing on hydrophobic cage-like decakis(methacryloxypropyl) silsesquioxane $\left(\mathbf{T}_{10}\right)$ and the linear analog PDMA- $b$-PMA- $b$-PEDOT, to explore the mechanism of SQ-based star configuration and conjugated system generating the efficient dispersion of SWNTs.

\section{Experimental Section}

2.1. Materials. N,N-(Dimethylamine)ethyl methacrylate (DMA, Aladdin, 99\%) and methyl acrylate (MA, Aladdin, 99\%) were purified through passing over the basic aluminum oxide column. 2,2-Azobisisobutyronitrile (AIBN, Aladdin, 98\%) was recrystallized twice in ethanol before use. Cagelike decakis(methacryloxypropyl) silsesquioxane $\left(T_{10}\right)$ was prepared in our laboratory [25]. Single-walled carbon nanotubes (SWNTs) were purchased from Aladdin (carbon content $>95 \%$, OD $\times L: 1-2 \mathrm{~nm} \times 5-30 \mu \mathrm{m})$.

2.2. Synthesis Processes. As described in Scheme 1, the experiments began from the precise synthesis of a $\mathbf{T}_{\mathbf{1 0}^{-}}$ (PDMA-b-PMA)-Th polymer precursor (M5-5) that contained five thiophene groups. The polymer precursor was subsequently copolymerized with the EDOT monomer to form PEDOT branches grafted from the hydrophobic $\mathbf{T}_{10}$ core, and the extension of the conjugated system was realized at the interface between the immiscible organic and aqueous phases by a modified interfacial synthesis protocol (MIP) [24]. For comparative purposes and to further explore the dispersing efficiency, the linear analog was prepared by the RAFT method and subsequently grafted the EDOT monomer from the thiophene group (denoted as PDMA- $b$-PMA- $b$-PEDOT) in the MIP process.

The specific experimental procedures were described in supporting information with structural characterization information for all polymers and reaction intermediates.

2.3. Characterization. Size exclusion chromatography (SEC) was conducted at $35^{\circ} \mathrm{C}$ by a Waters 1515 series system equipped with Styragel HR4 and HR3 columns and a Waters 2414 refractive index detector. The system was calibrated by narrowly dispersed polystyrene standards, and HPLC-grade DMF was used for the mobile phase at $0.60 \mathrm{~mL} / \mathrm{min}$. Nuclear Magnetic Resonance ( ${ }^{1} \mathrm{H}$ NMR) spectra were characterized in deuterated chloroform by using a Bruker DMX-400 spectrometer with a Varian probe. Infrared spectra were obtained with a Fourier transform infrared spectrometer using the KBr method (FTIR, Bruker Tensor 27, Germany). The UV-vis spectra of samples were measured in THF solution (UV-1800, Shimadzu Co., Japan). Weight loss temperatures of the products were determined by using a thermogravimetric analyzer (TGA, Netzsch TG209F3) at $10^{\circ} \mathrm{C} / \mathrm{min}$ under the nitrogen flow rate of $40 \mathrm{~mL} / \mathrm{min}$ from $50-700^{\circ} \mathrm{C}$. Transmission electron microscopy was performed on a high-resolution transmission electron microscope (HRTEM, Tecnai G2 F20 S-TWIN). The electrochemical performance was determined with CHI660E employing a standard three-electrode electrochemical cell that consisted of a glassy carbon electrode as the working electrode, a saturated calomel electrode (SCE) as the reference electrode, and a platinum wire as the counter electrode. Cyclic voltammetry was carried out relative to an SCE reference electrode at different scan rates $(20-100 \mathrm{mV} / \mathrm{s})$, and the potential window of cycling was confined between -1 and $1 \mathrm{~V}$. The electrochemical impedance spectroscopy (EIS) was performed in the frequency range of $10^{5}$ to $10^{-2} \mathrm{~Hz}$ with the signal amplitude of $5 \mathrm{mV}$ against the open-circuit potential. The electrical conductivity was subjected to the standard four-point probe method at ambient temperature (RTS-8, Four Probes Tech). 


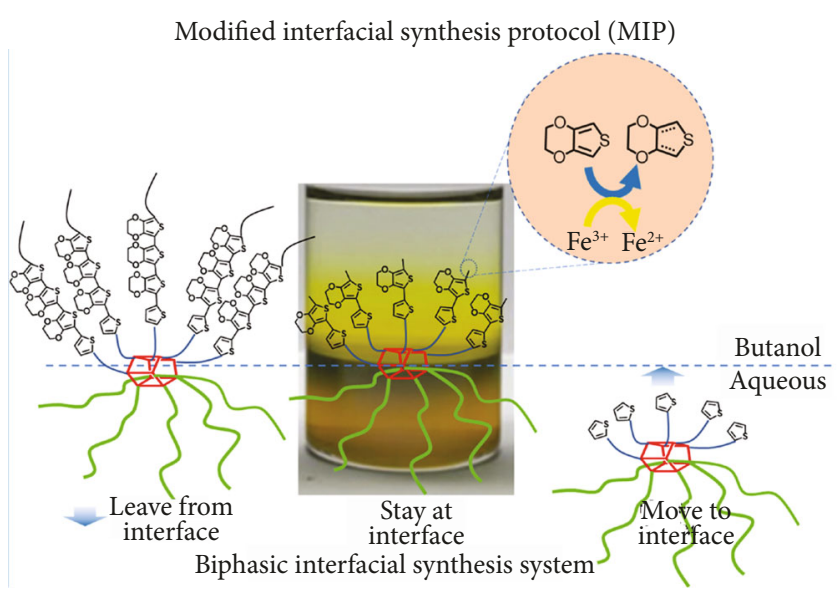

SCHEme 2: Synthesis protocol of P5-5 through water/butanol interface.

\section{Results and Discussion}

3.1. Chemical Structural Characterization. Decafunctionalized SQ $\left(\mathrm{T}_{10}\right)$ was selected to construct the star-shaped copolymer because this viscous amorphous liquid cluster compound was synthesized with high yield, which could be afforded in large-scale use. In addition, $\mathrm{T}_{10}$ offers more available functionalities for multiple grafting of the polymer chains to the core, and it also effectively interferes in strong $\pi-\pi$ interactions between neighboring conjugated chains, resulting in solution processability for the hybrid polymer [24].

In order to form the star-shaped copolymer precursor M5-5, a one-pot continuous reaction sequence was used to prepare the asymmetric $\mathrm{T}_{10}$ with five thiophene groups $\left(\mathrm{T}_{10}(5-5)\right)$, including a nucleophilic opening of the thiolactone by aminolysis and then a radical thiol-ene conjugation. Due to the extremely high reactivity of thiyl radical addition toward the carbon-carbon double bond, excess thiolactone could promote the conversion rate of amine and ensure complete conversion [26]; PDMA- $b$-PMA obtained by reversible addition-fragmentation transfer (RAFT) polymerization was linked to $\mathrm{T}_{10}(5-5)$ by thiol-ene conjugation after nucleophilic cleavage of the thiocarbonyl group.

The linear copolymer precursor was obtained by a typical RAFT polymerization procedure under the regulation of DTTC-Th as an efficient RAFT agent with an end-capped thiophene group.

Our particular synthetic approach (MIP) for the growth of conjugated chains relied on the reaction of the precursors and monomer by multicomponent oxidative coupling at the interface, which suppresses the interdiffusion of a monomer and an oxidant in the confined region to avoid uncontrolled homopolymerization, and also on the diffusion of the resulting copolymer down to the bottom phase due to the highly hydrophilic nature of the flexible arms and instability enhancement at the interface as the extension of the conjugated system, while being convenient for the rearrangement of unreacted precursors at the interface (Scheme 2).

${ }^{1} \mathrm{H}$ NMR, FTIR, UV-vis, and GPC analyses confirmed that the star-shaped conjugated copolymer and linear ana- logs were obtained with precise structure and low narrow molecular weight distribution, and the supporting information described the detailed experimental process and results (Figures S1-S8). All chemical structures are illustrated in Scheme 1, and the structural data are listed in Table 1.

3.2. Functionalized CNTs with the SilsesquioxanePolythiophene Hybrid Copolymer and Its Linear Analog. Multiple modifier-functionalized SWNTs were obtained while avoiding inclusion of dissociative copolymers through multiple centrifugation and washing steps, yielding PDMA- $b$ PMA-Th with SWNTs (M1) and PDMA- $b$-PMA- $b$-PEDOT with SWNTs (M2) and P5-5 with SWNTs. As we know, $\pi$ $-\pi$ stacking interactions are strong interfacial noncovalent interactions between modifiers and CNTs, as it was indicated that conjugation groups located at the flexible chains of the copolymer resulted in higher adhesion [8]. For the copolymer/CNT composites, the employed thiophene moieties in the flexible PDMAEMA could endow the copolymer strong $\pi-\pi$ interactions with CNT, functioning as interfacial linkers between the modifiers and SWNTs. Thus, the anchor groups at the flexible chains of modifiers could be adhered tightly along the CNT surface to form stronger interfacial interaction in the modifier-CNT complex.

3.2.1. Transmission Electron Microscopy (TEM). For the purpose to confirm the strong and stable interfacial interactions between SWNTs and modifiers, we conducted transmission electron microscopy (TEM) analysis. As observed in Figure 1, all nanotubes retained their structural integrity, revealing that no damage was evident to the nanotube walls in the experiment; the modified SWNTs apparently tended to exist as individuals or small bundles, especially for P55/SWNTs, despite well-known property that the pristine SWNTs would aggregate and be entangled to form large ropes without any modification. In addition, these starshaped copolymer-modified SWNTs had more individually dispersed nanotubes and less bundles, compared to linear copolymer-modified SWNTs; moreover, the star-shaped copolymer aggregates homogeneously adhered to the surface of the nanotube without large agglomeration. From the figures of individual SWNTs (Figure 1, insets), many arc-like bulges were observed on the surface of P5-5 modified nanotubes, attributed commonly to regions of irregular copolymer coating along the lengths; however, many linear copolymermodified nanotubes were still bounded together, which might be due to the disorderly stacking of a large copolymer and resulting in the lack of relatively strong noncovalent forces to stabilize individual tubes. Of the two linear copolymer-modified nanotubes, PDMA- $b$-PMA- $b$-PEDOTmodified SWNTs had less entanglement (Figure 1(b)).

3.2.2. Fourier Transform Infrared Spectroscopy (FTIR). Direct evidences of modifier-nanotube interactions could also be obtained using Fourier transform infrared spectroscopy (FTIR) after dispersion and centrifugation. As displayed in FTIR spectra of pristine SWNTs and modified SWNTs (Figure 2), the IR absorption bands occurred with maxima around $3450 \mathrm{~cm}^{-1}$, which correspond to the $-\mathrm{OH}$ stretching 
TABLE 1: Structural characterization of all copolymers.

\begin{tabular}{|c|c|c|c|c|c|c|c|c|c|c|}
\hline Polymer & $\begin{array}{l}\text { Yield } \\
(\%)\end{array}$ & $\begin{array}{c}M_{\mathrm{w}}{ }^{\mathrm{a}} \\
(\mathrm{kg} / \mathrm{mol})\end{array}$ & $M_{\mathrm{w}} / M_{\mathrm{n}}$ & $\begin{array}{c}M_{\mathrm{n}}{ }^{\mathrm{b}} \\
(\mathrm{kg} / \mathrm{mol})\end{array}$ & $\begin{array}{c}M_{\mathrm{n}}{ }^{\mathrm{c}} \\
(\mathrm{kg} / \mathrm{mol})\end{array}$ & $N_{\text {(thiophene) }}$ & $N_{\left(M_{\mathrm{n}}\right)}$ & $N_{\left(M_{\mathrm{w}}\right)}$ & $\mathrm{DP}_{\mathrm{EDOT}}^{\mathrm{b}}$ & $\mathrm{DP}_{\mathrm{EDOT}}^{\mathrm{a}}$ \\
\hline PDMA- $b$-PMA & 65 & 17 & 1.2 & 15 & 15 & - & - & - & - & - \\
\hline PDMA- $b$-PMA-Th & 65 & 21 & 1.3 & 20 & 20 & 1 & - & - & - & - \\
\hline $\begin{array}{l}\text { PDMA- } b \text {-PMA- } b- \\
\text { PEDOT }\end{array}$ & 70 & 22 & 1.3 & 21 & 21 & 1 & - & - & $6 / 6$ & $6 / 6$ \\
\hline M5-5 & 75 & 81 & 2.2 & 78 & 80 & 5 & 5.1 & 4.6 & - & - \\
\hline P5-5 & 50 & 91 & 2.2 & 92 & - & - & - & & $85 / 17$ & $70 / 14$ \\
\hline
\end{tabular}

${ }^{a}$ Molecular weights and polydispersity indexes determined by SEC in DMF on the basis of polystyrene calibration. The number of grafted EDOT and the approximate polymerization degree of EDOT in each PEDOT chain were calculated on SEC results. ${ }^{\mathrm{b}} M_{\mathrm{n}}$, the number of grafted EDOT, and the approximate polymerization degree of EDOT in each PEDOT chain were calculated on ${ }^{1} \mathrm{H}$ NMR results. ${ }^{c} M_{\mathrm{n}}$ was calculated on speculative structure construction. ${ }^{\mathrm{d}}$ The grafting number of PDMA- $b$-PMA in macromonomer precursors was calculated by the equation $N_{\left(M_{\mathrm{n}}\right)}=M_{\mathrm{n}}(\mathrm{macromonomer}$ precursor $) / M_{\mathrm{n}}$ (PDMA- $b$-PMA). ${ }^{\mathrm{e}}$ The grafting number of PDMA- $b$-PMA in macromonomer precursors was calculated by the equation $N_{\left(M_{\mathrm{w}}\right)}=M_{\mathrm{w}}($ macromonomer冈precursor) $/ M_{\mathrm{W}}$ (PDMA- $b$-PMA). ${ }^{\mathrm{f}}$ The number of thiophene rings of macromonomer precursors was calculated on ${ }^{1} \mathrm{H}$ NMR results.

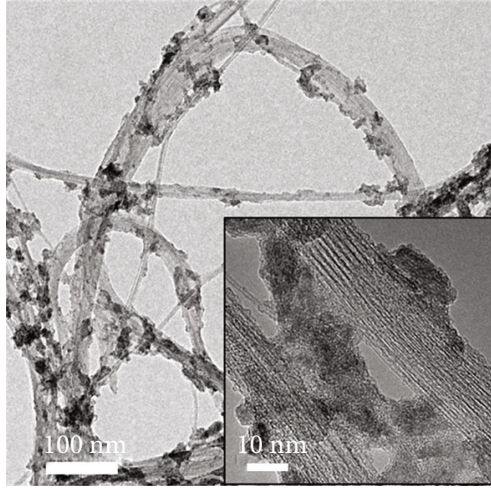

(a)

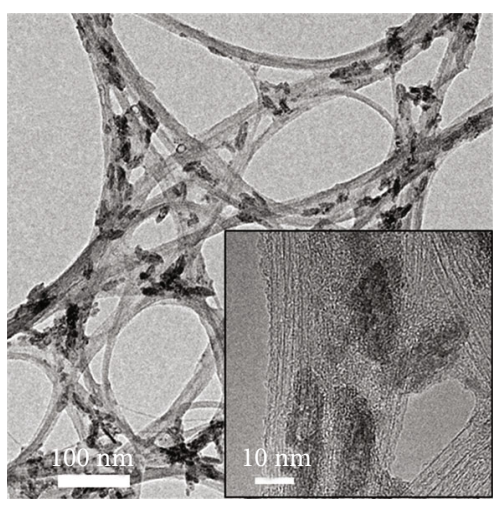

(b)

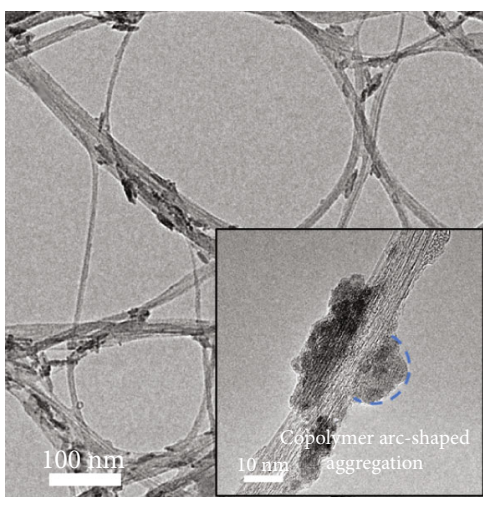

(c)

Figure 1: HR-TEM images of the modified SWNTs: PDMA- $b$-PMA-Th/SWNTs (a), PDMA- $b$-PMA- $b$-PEDOT/SWNTs (b), and P5$5 /$ SWNTs (c). The insets show the morphology of PDMA- $b$-PMA-Th, PDMA- $b$-PMA- $b$-PEDOT, and P5-5 surrounding the surface of the CNTs in (a), (b), and (c), respectively.

vibration of surface hydroxyl groups on CNTs and the adsorbed water molecules. An intense band at $1636 \mathrm{~cm}^{-1}$ appeared in all samples was assigned to the stretching vibration of carbonyl groups $(\mathrm{C}=\mathrm{O})$ present in carboxylic acids and conjugated $\mathrm{C}=\mathrm{C}$ groups on the nanotubes. Besides, in the spectra of modified SWNTs, an obvious $\mathrm{C}=\mathrm{O}$ stretching vibration of ester groups in polymer chains (ca. $1718 \mathrm{~cm}^{-1}$ ) and $\mathrm{C}-\mathrm{O}$ stretching vibrations (ca. 1233 and $1149 \mathrm{~cm}^{-1}$ ) were observed; the observation suggested that the modifiers were still effectively wrapped onto the nanotube surface by noncovalent interactions after rinsing. Compared to the spectra of modifiers (Figure S9), the characteristic peak of PEDOT chains (ca. 850 and $988 \mathrm{~cm}^{-1}$ ) shifted toward higher wavenumbers due to the electron-deficient defects of the nanotube surface and might confirm the $\pi$-conjugated stacking between the PEDOT segment and the nanotube surface, revealing the adherence of copolymers to the nanotube surface.

\subsection{Dispersion Properties}

3.3.1. UV-vis Spectrogram. For the assessment of the variation of dispersion efficiency of SWNTs modified by the star-like copolymer and its linear analogs in aqueous solution, three modified-SWNT (m-SWNT) dispersions (M1, M2, and M3) were prepared and investigated by using a UV-vis spectrogram. Figure S10 shows the absorption profiles of SWNT dispersions in a modifier THF/water solution; nearly no obvious signal peaks could be detected for SWNT dispersion modified by linear copolymers (M1, $\mathrm{M} 2$ ) in the UV-vis region. Moreover, there were no evident changes in the absorption spectra with or without the modifiers, causing us to tentatively suppose that the nanotube structure had not been destroyed in the solubilization process. In the absorption profiles for PDMA$b$-PMA- $b$-PEDOT solution and P5-5 solution (Figure 3 ), a wide absorption band from 450 to $550 \mathrm{~nm}$ was observed in the spectra, which could be attributed to the characteristic $\pi-\pi^{*}$ transition in conjugated polythiophene backbones [27].

Furthermore, we presumed that the amount of the nanotube stabilized by modifiers in dispersion would be reduced after centrifugal separation, and the UV absorption intensity of supernatants could represent the amount of well-stable nanotube suspension. As indicated from Figure 3, the absorption intensity of the supernatants for both PEDOT- 


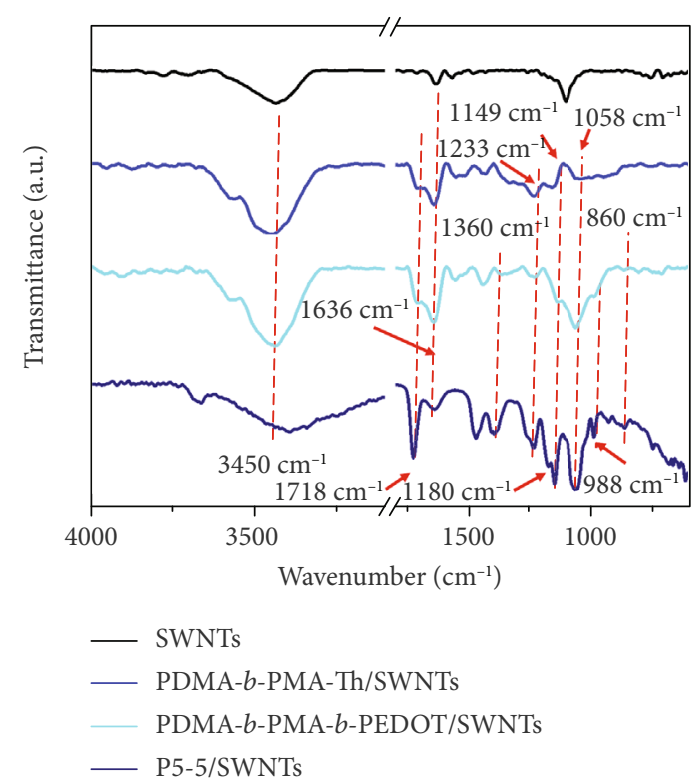

FIGURE 2: Infrared spectrogram of pristine SWNTs and modified nanotubes.

grafted modifiers (M2 and M3) was enhanced significantly in comparison with the original polymer solutions diluted in the same ratio, despite the fact that it was not obvious in comparison with the M1 supernatant with PDMA- $b$-PMA-Th solution; in addition, the M3 supernatant had the maximum enhancement of absorption intensity. The variation of dispersion efficiency resulted from the noncovalent modification, which was probably relevant to the configuration, the silsesquioxane cage, and the PEDOT segments.

3.3.2. Visual Sedimentation. It was well known that pristine SWNTs could not be stabilized in aqueous solution without the help of a modifier, despite the vigorous ultrasonication, due to the highly specific surface area and hydrophobic sidewall surface. For the modified SWNTs, the modification ability of polymers on SWNTs could be visually assessed by conducting a sedimentation experiment to compare the stability of modified-SWNT dispersions (Figure S11). All modifiers had the ability to enhance the dispersity of SWNTs, especially PEDOT-grafted copolymers. In particular, it seemed that the star-shaped copolymer/SWNT dispersion had more stability than linear ones, which was observed in a vial over 10 days without any precipitation. Therefore, we inferred that the SQ cage and PEDOT segments played key roles for noncovalent functionalization of nanotubes: Firstly, due to the hydrophobic nature of SQ cores and PEDOT segments, they should be gathered close to the surface of the carbon tube. Secondly, the poor solubility and conjugated nature of PEDOT imparted effective physisorption between the stabilizer and the conjugated structure of the nanotube. Thirdly, thanks to the tentacles of multiarm SQ-based copolymers with rigid arms, the solvation segments would produce more opportunities to generate a great affinity for solvent, which primarily decreased the interfacial tension of nanotubes by minimizing their contacts with water [28],

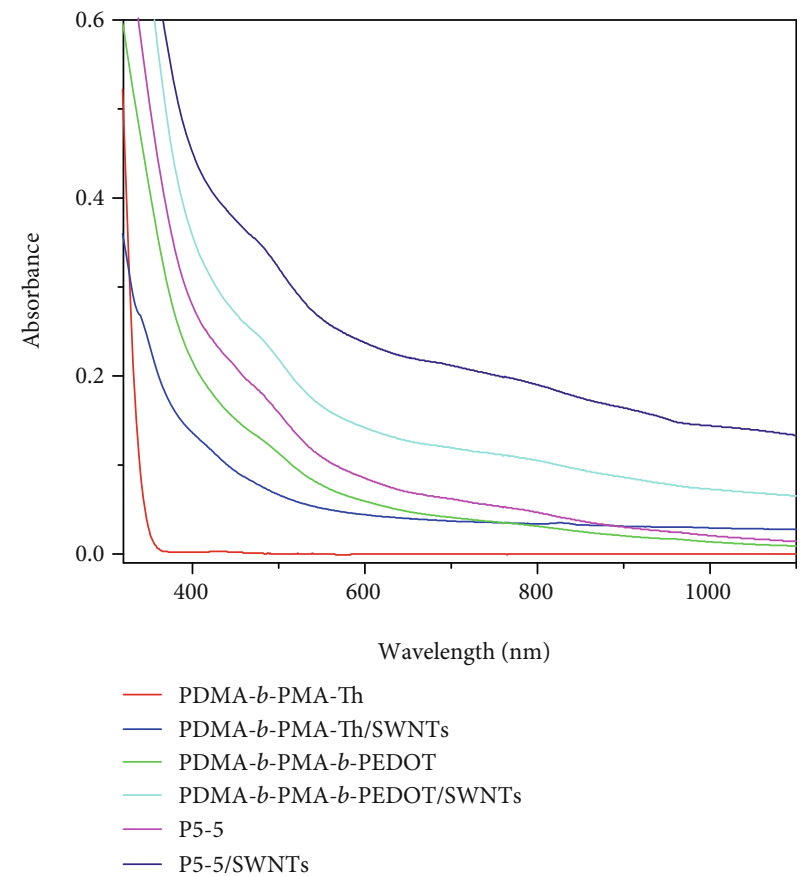

FIGURE 3: Absorption profiles for the modifier solution and supernatant of SWNT dispersion. The supernatant was obtained by merging the centrifugal supernatant and the washing liquid (the total volume of washing liquid was four times as much as the volume of dispersion), and all copolymer solutions were diluted to $1 / 5$ of their original concentration [4].

and more hydrophobic segments could provide more opportunities to interact with the nanotube surface. This speculation can be strongly supported by the fact that the SQ-based star copolymer employing multi-PEDOT chains compared to its linear analogs resulted in an improved functional capability to stabilize SWNTs.

3.3.3. Thermogravimetric Analysis (TGA). For the purpose of obtaining a more quantitative picture of the extent of nanotube functionalization, thermogravimetric analysis (TGA) was conducted to evaluate the thermodynamic property of copolymer/SWNT complexes and further estimate the well-adhered polymers on the nanotube surface under an $\mathrm{N}_{2}$ atmosphere after centrifugation; all thermal property data are listed in Table 2 . The copolymers (PDMA- $b$-PMA-Th, PDMA- $b$-PMA- $b$-PEDOT, and P5-5) and copolymer/SWNTs complexes (M1 residue, M2 residue, and M3 residue) (Figure 4) both indicated a distinct two-step thermal degradation, which might be caused by the loss of ester bonds and the decomposition of the backbone in the temperature range of $200-500^{\circ} \mathrm{C}$, while no mass loss of the SWNTs was observed in the same temperature range.

The highest decomposition temperature at the maximum degradation rate in the second stage for thermal degradation of the framework structure of P5-5 indicated that the starshaped configuration and the presence of $\mathrm{T}_{10}$ contributed to the increase in thermostability (Figure S12) [29]. 
TABLE 2: Thermogravimetric analysis data of various copolymers and modified-SWNT residues.

\begin{tabular}{lccc}
\hline & $T_{\max , 1}\left({ }^{\circ} \mathrm{C}\right)$ & $T_{\max , 2}\left({ }^{\circ} \mathrm{C}\right)$ & $C_{700}{ }^{\mathrm{a}}(\%)$ \\
\hline $\mathrm{T}_{10}$ & 418 & - & 43.7 \\
PDMA- $b$-PMA- $b$-Th & 325 & 421 & 5.5 \\
PDMA- $b$-PMA- $b$-PEDOT & 320 & 406 & 5.1 \\
P5-5 & 307 & 430 & 12.2 \\
PEDOT & 325 & 375 & 30.4 \\
M1 residue & 281 & 398 & 72.2 \\
M2 residue & 280 & 402 & 60.4 \\
M3 residue & 295 & 412 & 56.1 \\
\hline
\end{tabular}

${ }^{\mathrm{a}} \mathrm{C}_{700}$ represented the percentage residue of various samples at $700^{\circ} \mathrm{C}$.

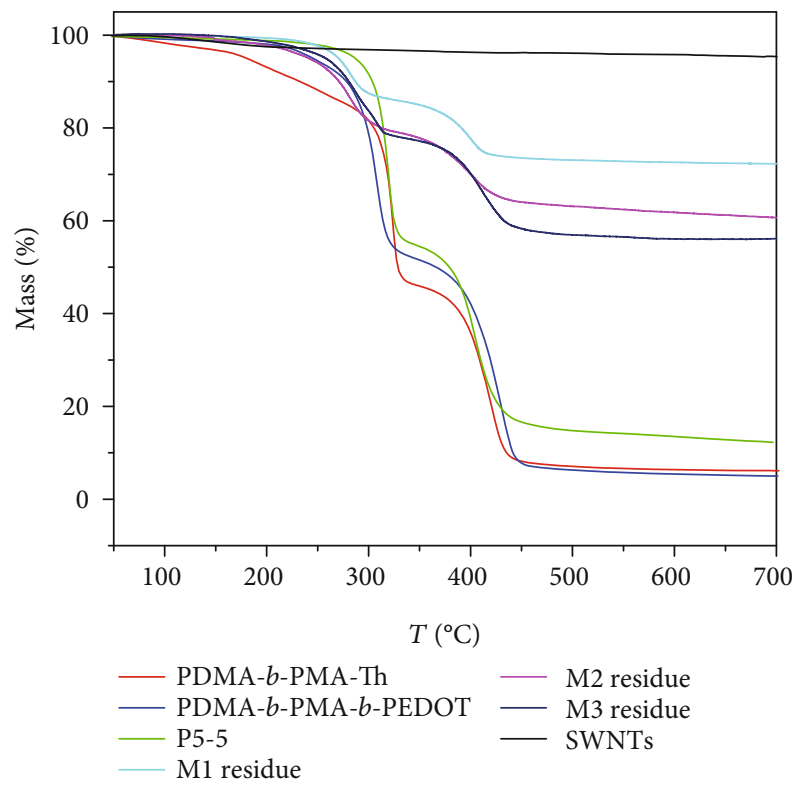

FIGURE 4: TGA curves of the modifiers, modified-SWNT residues, and pristine SWNTs.

The lower decomposition temperature of the PEDOT homopolymer might result in the lower decomposition temperature in the second stage of the PDMA- $b$-PMA- $b$ PEDOT trace (Figure S13), and the successful grafting of PEDOT segments from the thiophene group of PDMA- $b$ PMA-Th was further verified.

In addition, the $C_{700}$ values of PDMA- $b$-PMA-Th, PDMA- $b$-PMA- $b$-PEDOT, and P5-5 were $5.5,5.1$, and $12.6 \%$, and the $C_{700}$ values of $M 1$ residue, $M 2$ residue, and M3 residue were 72.2, 60.4, and 56.1, respectively. As indicated in Figure 3 (TGA traces), the copolymers would be completely decomposed over $500^{\circ} \mathrm{C}$; the mass loss from the residues of copolymer/SWNT complexes during thermal degradation could be used to estimate the amount of well-wrapped copolymers on the nanotubes in dispersion, and it was, respectively, about $25 \%, 35 \%$, and $44 \%$ corresponding to the loss of PDMA$b$-PMA-Th, PDMA- $b$-PMA- $b$-PEDOT, and P5-5 between $200^{\circ} \mathrm{C}$ and $500^{\circ} \mathrm{C}$ deducing that the mass loss of SWNTs, further combined with UV spectra, could be used to evaluate the functional capability of modifiers to stabilize SWNTs.
M1 residue had the least mass loss with the lowest dispersion efficiency of M1; that is, most of PDMA- $b$-PMA-Th was washed out due to the unstable physisorption on SWNTs; it implied that the grafting of PEDOT segments played an important role to form stable interactions with nanotubes in solution, which endowed more stacking interaction and hydrophobicity between modifiers and the surface of the nanotube. As expected, M3 residue had the largest mass loss compared with the linear ones with the best dispersion efficiency of M3, indicating that more P5-5 was well wrapped on the surface of nanotubes resulting in the largest polymer content in residue, thus providing strong support for the previous proposal: more possibilities would be obtained by introducing more solvation segments to generate a great affinity for solvent, and more hydrophobic segments and conjugated segments could provide more opportunities and $\pi$-stacking to interact with the nanotube, which powerfully enhanced adhesion on the surface to avoid dissolving even during several washing steps.

3.3.4. Mechanism. On the basis of the above results, the possible mechanism for elucidating the interaction between SWNTs and copolymers in Figure 5 was put forward. Thanks to the hydrophobic interaction and $\pi-\pi$ stacking system between star-shaped copolymers and the sidewall, originating from the hydrophobic CSQ cores and conjugated PEDOT segments, the modifiers were readily adsorbed onto the surface of nanotubes by forming relatively strong noncovalent forces, and the other flexible solvable tentacles on the opposite side of the carbon tube had to stretch to the solvent as linear "buoys" due to the steric hindrance endowed by silsesquioxane cages. Thereby, a mass of spherical modifiers was inclined to absorb onto the sidewall to form arc-shaped copolymer aggregation which could decrease the hydrophobicity of the sidewall for minimizing the direct contact with water and other tubes. Meanwhile, these "buoys" tremendously enhanced the solubility of SWNTs in aqueous solution, thus causing well-dispersed SWNTs. In contrast, linear modifiers would be irregularly distributed in solvent due to lack of restrictions from the CSQ cage; therefore, the solvable copolymer chains would wrap the nanotube by generating hydrogen bond interactions. Although the PEDOT chains in PDMA- $b$-PMA- $b$-PEDOT could provide some $\pi-\pi$ stacking effect to enhance adhesive force with the graphite structure of the surface, but the linear copolymers were difficult to form well-organized aggregations to prevent bundling of tubes.

3.4. Electrochemical Property. The electrochemical characteristics of thin films prepared by spin coating from copolymer solutions and the supernatant of modified-SWNT dispersions were determined to provide information about the structure of copolymers and dispersion efficiency. The quasi-rectangle-shaped cyclic voltammetry $(\mathrm{CV})$ curves of PDMA-PMA- $b$-PEDOT P5-5 in Figure S14 were similar to those of soluble PEDOT analogs [30], the curves indicated similar chemically reversible signals during the process of positive and negative scans, and low potentials of the current onsets verified the highly electron-rich 


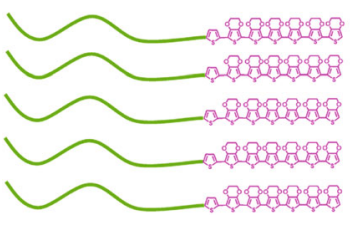

Linear conjugated copolymers

$\sim$ :PDMA- $b$-PMA chain

B : Cage of silsesquioxane

$\sim$ : PEDOT segments

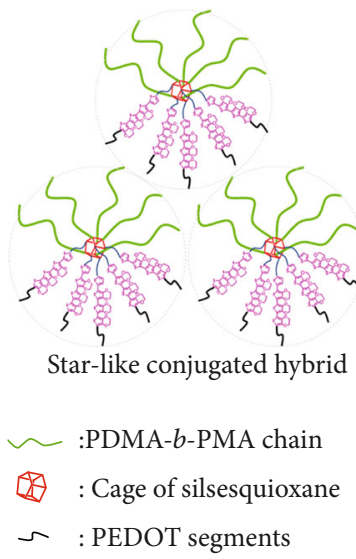

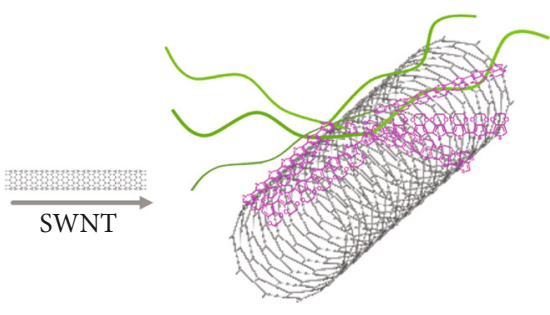

Copolymer-nanotube complex

(a)

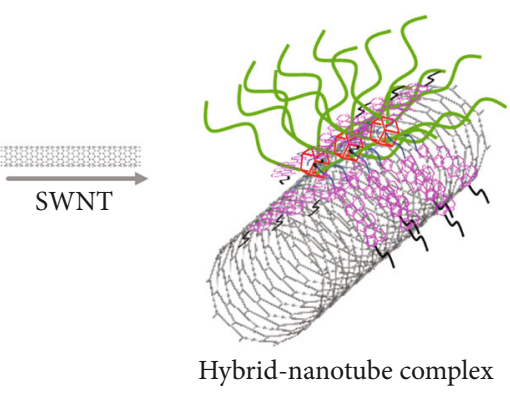

Hybrid-nanotube complex

(b)

FIgURe 5: Mechanism between SWNTs with modifiers.

nature of the polymer films. As shown in the CV curves, a negative shift was observed in the oxidation potential $\left(E_{\mathrm{ox}}\right)$ values for PDMA- $b$-PMA- $b$-PEDOT $(0.84 \mathrm{~V})$ and P5-5 $(0.75 \mathrm{~V})$, possibly due to the larger conjugation system in $\mathrm{P} 5-$ 5. In addition, the larger curve area in the P5-5 film indicated that it had a higher specific capacitance that was imparted by the more PEDOT chains for facilitating ion motion.

In order to further study the stabilizing effect endowed by the conjugation and configuration of modifiers, which could be reflected by the electrochemical variation of modifiedSWNT composite films, cyclic voltammetry (CV) and electrochemical impedance spectroscopy (EIS) measurements were conducted on the composite films. From Figure 6(a), CV curves of the P5-5/SWNT composite film and PDMA$b$-PMA- $b$-PEDOT/SWNT composite film showed a quasirectangle shape with a couple of peaks, indicating an electrical double-layer capacitance originated from the redox reactions of PEDOT chains [31]. As we know, a large surface area and good electrical conductivity may enhance capacity properties, and the curve area of the P5-5/SWNT composite film was greater than that of the PDMA- $b$-PMA- $b$-PEDOT/SWNT composite film and PDMA- $b$-PMA-Th/SWNT composite film; it was clearly indicated that the hybrid copolymer, P5-5, possesses maximum PEDOT segments and star configuration, which was beneficial to facilitating ion motion to increase the specific capacitance of the composite film.
Furthermore, the internal resistance of modified-SWNT films, arising from the physical contact between components and the electron transfer resistance between the active materials and the substrate, could reflect the adhesion and $\pi-\pi$ stacking system between copolymers and the nanotube [32-34]. Therefore, electrochemical impedance spectroscopy (EIS) measurements were performed from $10^{5} \mathrm{~Hz}$ to $0.01 \mathrm{~Hz}$. As shown in Figure $6(\mathrm{~b})$, the resistance of the electrolyte $\left(R_{\mathrm{sol}}\right)$ corresponded to the intercept of the Nyquist diagrams on the $Z$ real axis; the charge transfer resistance $\left(R_{\mathrm{ct}}\right)$ on the electrode was represented on the diameter of the semicircle in the Nyquist plots; the Warburg resistance $\left(R_{\mathrm{W}}\right)$ was associated with the slope of the straight line portion in the Nyquist plots at low frequency, correlating with the frequency dependence of ion diffusion to the electrode interface in the electrolyte. Obviously, $R_{\text {sol }}$ values for the P5-5/SWNT composite film (12.7 $\Omega$ ), PDMA- $b$-PMA$b$-PEDOT/SWNT composite film (12.9 $\Omega$ ), and PDMA- $b$ PMA-Th/SWNT composite film (13.4 $\Omega$ ) were quite similar for the same electrochemical system. Predictable results, in which $R_{\mathrm{ct}}$ values were in the order of the P5-5/SWNT composite film $(76 \Omega)<$ PDMA- $b$-PMA- $b$-PEDOT/SWNT composite film $(116 \Omega)<$ PDMA- $b$-PMA-Th/SWNT composite film $(>186 \Omega)$, indicated that PEDOT chains and silsesquioxane cages tended to assist the charge migration, while further implying that the star-shaped copolymer could stabilize more nanotubes via more $\pi-\pi$ interaction and hydrophobic- 


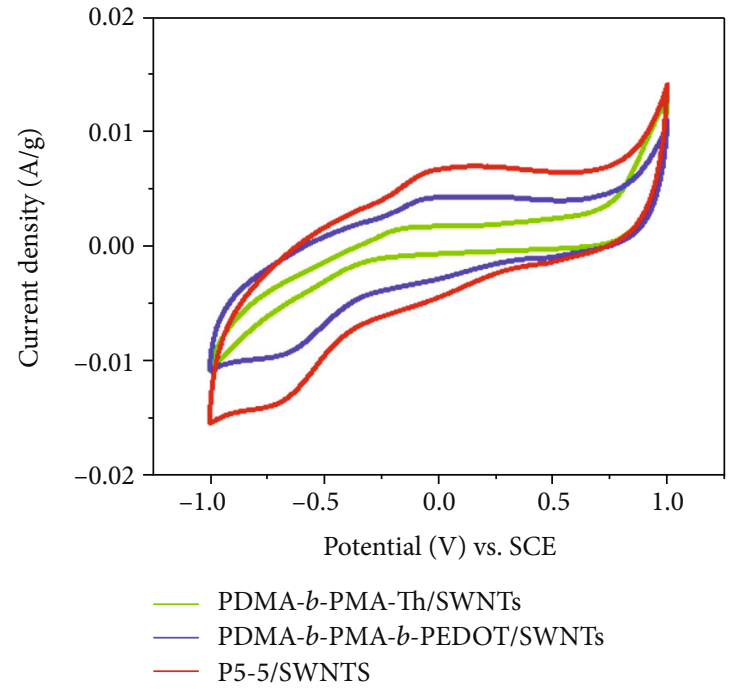

(a)

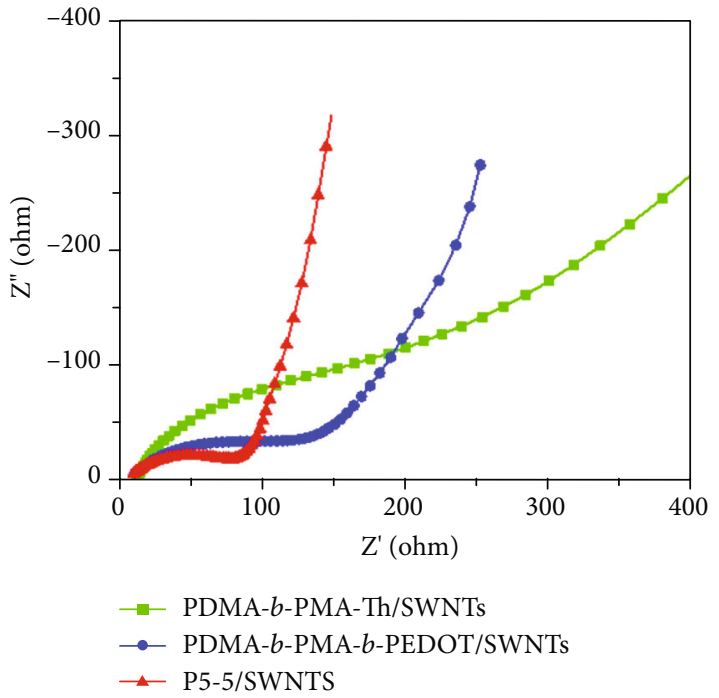

(b)

Figure 6: Cyclic voltammograms of the copolymer/SWNT composite at $50 \mathrm{mV} / \mathrm{s}$ (a) and electrochemical impedance spectroscopy of the copolymer/SWNT composite (b).

hydrophobic interaction. The $R_{\mathrm{w}}$ value for the P5-5/SWNT composite film was larger than that of PDMA- $b$-PMA- $b$ PEDOT/SWNTs and PDMA- $b$-PMA-Th/SWNTs, which demonstrated good accessibility of the electrolyte ions to the P5-5/SWNT electrode in the participation of robust silsesquioxane cages on the surface. These EIS results supported the CV analyses of the enhanced electrochemical performance of the P5-5/SWNT composite film and also echoed the analyses of UV spectra and TEM; that is, the SQ-based star-shaped copolymer with PEDOT segments could tightly adhere on the surface to stabilize more nanotubes in aqueous solution.

\section{Conclusion}

In summary, one star-shaped silsesquioxane-polythiophene hybrid modifier (P5-5) for SWNTs was successfully synthesized via a modified interfacial synthesis protocol. The visual sedimentation indicated that the P5-5 showed excellent ability to stabilize SWNTs in aqueous solution, and direct evidences of modifier/SWNT interactions were verified by UV-vis, FTIR, TEM, and TGA, which demonstrated that the amphiphilic hybrid copolymer was much more efficient at modifying SWNTs than its linear analogs. The possible mechanism for elucidating the noncovalent interaction between SWNTs and the star-shaped copolymer was that the silsesquioxane core and conjugated PEDOT chains locally anchored onto the nanotube surface due to their hydrophobicity nature, while the flexible copolymer chains stretched into aqueous solution; in addition, the formation of amphiphilic copolymer aggregations, which is convex over the sidewall surface, sequentially reduced the hydrophobic area for minimizing their contact with water and other tubes and thus provided hydrophilic "buoys" to enhance the solubility of SWNTs in aqueous solution. The electrochemical properties indicated that PEDOT chains and silsesquioxane cages tended to facilitate the charge migration due to the electrochemical activity and functional capability of P5-5.

\section{Data Availability}

The data used to support the findings of this study are included within the article.

\section{Conflicts of Interest}

The authors declare that they have no conflicts of interest.

\section{Authors' Contributions}

Shuxi Gao and Xiaoyong Hu contributed equally to this work.

\section{Acknowledgments}

The authors gratefully acknowledge the financial support from the China Postdoctoral Science Foundation (2019M662819) and the Guangdong Academy of Sciences Project of Science and Technology Development (2018GDASCX-1011) and the material support from Professor Kai Xu's group (Guangzhou Institute of Chemistry, Chinese Academy of Sciences).

\section{Supplementary Materials}

The only supplied supporting information consists of 14 pages (single column) including 14 figures. It contained the detailed description of the experimental process and some additional results for accurate argument. (Supplementary Materials)

\section{References}

[1] S. Iijima, "Helical microtubules of graphitic carbon," Nature, vol. 354 , no. 6348 , pp. 56-58, 1991. 
[2] R. H. Baughman, A. A. Zakhidov, and W. A. de Heer, "Carbon nanotubes-the route toward applications," Science, vol. 297, no. 5582, pp. 787-792, 2002.

[3] M. Yang, V. Koutsos, and M. Zaiser, "Interactions between polymers and carbon nanotubes: a molecular dynamics study," The Journal of Physical Chemistry B, vol. 109, no. 20, pp. 10009-10014, 2005.

[4] S. Gao, Z. Yu, K. Xu et al., "Silsesquioxane-cored star amphiphilic polymer as an efficient dispersant for multiwalled carbon nanotubes," RSC Advances, vol. 6, no. 36, pp. 30401-30404, 2016.

[5] A. Di Crescenzo, M. Aschi, and A. Fontana, "Toward a better understanding of steric stabilization when using block copolymers as stabilizers of single-walled carbon nanotubes (SWCNTs) aqueous dispersions," Macromolecules, vol. 45, no. 19, pp. 8043-8050, 2012.

[6] N. A. Rice and A. Adronov, "Supramolecular interactions of high molecular weight poly (2,7-carbazole) s with singlewalled carbon nanotubes," Macromolecules, vol. 46, no. 10, pp. 3850-3860, 2013.

[7] A. Habibnejad Korayem, M. R. Barati, G. P. Simon et al., "Transition and stability of copolymer adsorption morphologies on the surface of carbon nanotubes and implications on their dispersion," Langmuir, vol. 30, no. 33, pp. 1003510042, 2014

[8] K. Y. Cho, Y. S. Yeom, H. Y. Seo et al., "Rational design of multiamphiphilic polymer compatibilizers: versatile solubility and hybridization of noncovalently functionalized CNT nanocomposites," ACS Applied Materials \& Interfaces, vol. 7, no. 18, pp. 9841-9850, 2015.

[9] P.-C. Ma, N. A. Siddiqui, G. Marom, and J.-K. Kim, "Dispersion and functionalization of carbon nanotubes for polymerbased nanocomposites: a review," Composites Part A: Applied Science and Manufacturing, vol. 41, no. 10, pp. 1345-1367, 2010.

[10] H. Abbasi, M. Antunes, and J. I. Velasco, "Recent advances in carbon-based polymer nanocomposites for electromagnetic interference shielding," Progress in Materials Science, vol. 103, pp. 319-373, 2019.

[11] A. S. R. Bati, L. Yu, M. Batmunkh, and J. G. Shapter, "Recent Advances in Applications of Sorted Single-Walled Carbon Nanotubes," Advanced Functional Materials, vol. 29, no. 30, p. 1902273, 2019.

[12] M. W. Chik, Z. Hussain, M. Zulkefeli et al., "Polymer-wrapped single-walled carbon nanotubes: a transformation toward better applications in healthcare," Drug Delivery and Translational Research, vol. 9, no. 2, pp. 578-594, 2019.

[13] K.-t. Lau, C. Gu, and D. Hui, "A critical review on nanotube and nanotube/nanoclay related polymer composite materials," Composites Part B: Engineering, vol. 37, no. 6, pp. 425-436, 2006.

[14] A. S. Christensen, T. Kubař, Q. Cui, and M. Elstner, "Semiempirical quantum mechanical methods for noncovalent interactions for chemical and biochemical applications," Chemical Reviews, vol. 116, no. 9, pp. 5301-5337, 2016.

[15] Y. Zhou, R. Azumi, and S. Shimada, "A highly durable, stretchable, transparent and conductive carbon nanotube-polymeric acid hybrid film," Nanoscale, vol. 11, no. 9, pp. 3804-3813, 2019.

[16] Y.-L. Zhao, N. Ullah, S. Chen, and R.-Q. Zhang, " $n \rightarrow \pi^{*}$ interaction promoted charge carrier transfer between helical
SWNTs and a 4-(1-pyrenyl) phenyl group," The Journal of Physical Chemistry C, vol. 123, no. 22, pp. 13976-13982, 2019.

[17] P. Bilalis, D. Katsigiannopoulos, A. Avgeropoulos, and G. Sakellariou, "Non-covalent functionalization of carbon nanotubes with polymers," RSC Advances, vol. 4, no. 6, pp. 2911-2934, 2014.

[18] L. V. Sigolaeva, T. V. Bulko, M. S. Kozin et al., "Long-term stable poly(ionic liquid)/MWCNTs inks enable enhanced surface modification for electrooxidative detection and quantification of dsDNA," Polymer, vol. 168, pp. 95-103, 2019.

[19] G. Zhang, M. McBride, N. Persson et al., "Versatile interpenetrating polymer network approach to robust stretchable electronic devices," Chemistry of Materials, vol. 29, no. 18, pp. 7645-7652, 2017.

[20] Z.-H. Jin, Y.-L. Liu, J.-J. Chen, S.-L. Cai, J.-Q. Xu, and W.H. Huang, "Conductive polymer-coated carbon nanotubes to construct stretchable and transparent electrochemical sensors," Analytical Chemistry, vol. 89, no. 3, pp. 2032-2038, 2017.

[21] Y. Huang, Y. Wang, L. Gao, X. He, P. Liu, and C. Liu, "Characterization of stretchable SWCNTs/Lycra fabric electrode with dyeing process," Journal of Materials Science: Materials in Electronics, vol. 28, no. 5, pp. 4279-4287, 2017.

[22] L. Yu, C. Shearer, and J. Shapter, "Recent development of carbon nanotube transparent conductive films," Chemical Reviews, vol. 116, no. 22, pp. 13413-13453, 2016.

[23] I. A. Kinloch, J. Suhr, J. Lou, R. J. Young, and P. M. Ajayan, "Composites with carbon nanotubes and graphene: an outlook," Science, vol. 362, no. 6414, pp. 547-553, 2018.

[24] S. Gao, K. Xu, X. Gui, L. Sun, and L. Liu, "Synthesis of soluble silsesquioxane-polythiophene hybrid copolymers with a controlled morphology," ACS Applied Polymer Materials, vol. 1, no. 3, pp. 437-451, 2019.

[25] J. Peng, K. Xu, H. Cai et al., "Can an intact and crystalline octakis(methacryloxypropyl) silsesquioxane be prepared by hydrolysis-condensation of a trimethoxysilane precursor?," RSC Advances, vol. 4, no. 14, pp. 7124-7131, 2014.

[26] H. Willcock and R. K. O'Reilly, "End group removal and modification of RAFT polymers," Polymer Chemistry, vol. 1, no. 2, pp. 149-157, 2010.

[27] J. F. Ponder Jr., A. M. Österholm, and J. R. Reynolds, “Designing a soluble PEDOT analogue without surfactants or dispersants," Macromolecules, vol. 49, no. 6, pp. 2106-2111, 2016.

[28] Z. Iatridi and C. Tsitsilianis, "pH responsive MWCNT-star terpolymer nanohybrids," Soft Matter, vol. 9, no. 1, pp. 185193, 2013.

[29] S. Majeed, V. Filiz, S. Shishatskiy, J. Wind, C. Abetz, and V. Abetz, "Pyrene-POSS nanohybrid as a dispersant for carbon nanotubes in solvents of various polarities: its synthesis and application in the preparation of a composite membrane," Nanoscale Research Letters, vol. 7, no. 1, p. 296, 2012.

[30] H. Zhang, T.-T. Lu, W.-Y. Lai et al., "Pyrene-cored starburst oligofluorenes with diphenylamine end-cappers: design, synthesis, stabilized optical gain, and lasing properties," The Journal of Physical Chemistry C, vol. 121, no. 49, pp. 27569-27579, 2017.

[31] T. Goda, M. Toya, A. Matsumoto, and Y. Miyahara, "Poly(3,4ethylenedioxythiophene) bearing phosphorylcholine groups for metal-free, antibody-free, and low-impedance biosensors specific for C-reactive protein," ACS Applied Materials \& Interfaces, vol. 7, no. 49, pp. 27440-27448, 2015. 
[32] C. Wei, W. Zhang, Y. Zhou et al., "High-performance ternary $\pi$-conjugated copolymers containing diarylethylene units: synthesis, properties, and study of substituent effects on molecular aggregation and charge transport characteristics," Journal of Materials Chemistry C, vol. 7, no. 2, pp. 362-370, 2019.

[33] Z. Zhou, C. Gu, C. Chen, P. Zhao, Y. Xie, and J. Fei, “An ultrasensitive electrochemical sensor for quercetin based on 1-pyrenebutyrate functionalized reduced oxide graphene $/$ mercapto- $\beta$ - cyclodextrin /Au nanoparticles composite film," Sensors and Actuators B: Chemical, vol. 288, pp. 8895, 2019.

[34] P. Zhao, M. Ni, C. Chen et al., "Stimuli-enabled switch-like paracetamol electrochemical sensor based on thermosensitive polymer and MWCNTs-GQDs composite nanomaterial," Nanoscale, vol. 11, no. 15, pp. 7394-7403, 2019. 